\title{
les propriétés de déformation et de résistance des matériaux à gros grains
}

\author{
par \\ $D^{r}$ P. Anagnosti \\ Professeur à Belgrade, Yougoslavie
}

Le choix des dimensions et la prévision du comportement des ouvrages construits avec des matériaux à gros grains (approximativement $D_{50}>50 \mathrm{~mm}$; $D_{\max }<1000-2000 \mathrm{~mm}$ ), ou bien fondés sur des matériaux ả gros grains, imposent la connaissance de leurs propriétés mécaniques servant de base aux calculs. L'étude et la détermination des propriétés mécaniques des matériaux à gros grains rencontrent certaines difficultés en raison du grand volume et du poids des échantillons comportant tous les grains, de l'équipement spécial nécessaire au traitement des échantillons, ainsi que des dispositifs d'essais compliqués et très grands pour les essais.

D'autre part, le développement permanent des équipements de construction a abouti à des engins d'excavation (dragues à bennes de 5 à $10 \mathrm{~m}^{3}$ ) et de transport des matériaux (dumpeurs-camions basculants de 15 à $30 \mathrm{~m}^{3}$ ) permettant que l'utilisation de matériaux à gros grains pour les digues et barrages apparaisse comme une solution optimale aussi bien du point de vue de la vitesse que des paramètres économiques. C'est pourquoi, il n'est pas surprenant qu'au cours des dernières 15 à 20 années, on ait consacré de plus en plus d'attention à l'étude des propriètés de ces matériaux et à la rationalisation des dimensions des ouvrages constitués de tels matériaux tels que : remblais, déblais, digues, barrages, etc.

Actuellement aussi maintes recherches sont orientées vers la détermination des principales propriétés mécaniques de déformabilité et de résistance au cisaillement sur de petits échantillons (volume jusqu'à $1 \mathrm{~m}^{3}$ ) pouvant être fabriqués facilement en laboratoire et permettant des essais détaillés et précis. Toutefois, afin que les données obtenues sur de petits échantilIons puissent être appliquées au matériau réel, il faut définir les rapports de similitude du matériau du modèle, c'est-à-dire du matériau de l'échantillon et du matériau réel de l'ouvrage. La solution de ce problème de similitude avec le modèle est recherché par:

a) L'analyse différentielle ou élémentaire basée sur l'étude de l'influence de la géométrie des grains, de leur qualité, de l'interaction entre les grains d'après les mécanismes cinématiques et statiques (à savoir d'après les déplacements relatifs et les forces aux contacts), de l'assemblage des grains, de la porosité plus ou moins élevée, etc.

b) L'analyse globale basée sur l'étude de la dilatance, c'est-à-dire du changement du volume des échantillons lors du cisaillement, de l'importance du niveau de contrainte sur les aspects du travail dépensé irréversiblement lors de la déformation (écrasement et fragmentation des grains, frottement, déformation irréversible), de l'influence du mode du chargement et des conditions réelles de l'échantillon lors des essais.

Les conditions théoriques de similitude physique ne peuvent être satisfaites que si l'échantillon comporte des grains de composition pétrographique identique à celle du matériau réel, alors que la formation d'un échantillon qui comporterait des grains géométriquement semblables et des propriétés de déformation et de densité semblables ne peut pas encore être réalisée. C'est pourquoi, le problème est résolu par des essais sur échantillons, en faisant varier les paramétres prédominants tels que : grandeur des grains, niveau des contraintes normales (octaédriques), volume des échantillons, porosité et éventuellement assemblage des grains. Prenant en considération la variabilité relative de la composition du matériau à gros grains réalisée dans l'ouvrage par suite des variations de la qualité du matériau dans la carrière, du fait des méthodes et des effets du minage ou du défonçage (excavation sans minage), du mode de mise en ceuvre, etc.; la connaissance de la "sensibilité" aux effets provenant des modifications de certains paramètres mentionnés permet de déterminer les propriétés envisagées extrêmes et moyennes de la déformabilité et de la résistance au cisaillement pour le matériau réel. En fonction de l'importance et du but de l'ouvrage et des propriétés spécifiques du matériau, le nombre 
de paramètres examinés augmente ou diminue, conduisant ainsi à une marge de tolérance plus ou moins grande sur les propriétés mécaniques prévues pour le matériau réel.

La connaissance de la dépendance complexe des propriétés des matériaux à gros grains permet à l'ingénieur de choisir les essais permettant d'étudier les paramètres nécessaires et de connaître au moins approximativement l'influence qu'exercent les autres paramètres qui ne seront pas l'objet de recherches aussi détaillées.

L'objectif principal de cette étude est de présenter une idée complète du problème et de rendre plus facile la programmation pratique des essais et le choix des propriétés à retenir pour les matériaux à gros grains.

\section{Introduction}

Le problème traité dans cette étude existe déjà depuis longtemps dans la pratique de l'ingénieur. Lors de l'utilisation des engins mécaniques modernes de terrassement du rocher, la grandeur du " grain n peut atteindre un volume de $4 \mathrm{~m}^{3}$ (dimensions moyennes de 1,0 à $1,5 \mathrm{~m}$ ). Certains équipements de laboratoire permettent la confection et l'essai d'échantillons de $1 \mathrm{~m}^{3}$ dont les grains ne dépassent pas $20 \mathrm{~cm}$ et ceci uniquement dans quelques laboratoires spécialisés (U.S. A., Mexique, etc.). Dans la plupart des laboratoires la dimension maximale des échantillons est de l'ordre de 30 à $40 \mathrm{~cm}$, de telle sorte que les propriétés mécaniques des matériaux à gros grains ne peuvent pas être soumises directement aux essais de laboratoire. C'est d'autant plus regrettable qu'on constate souvent, pour des matériaux de grandeur variable de grains et de courbe granulométrique de même forme, que les matériaux à grains les plus gros ont une résistance au cisaillement moins élevée (Marsal 1973). D'autre part, on sait très bien que le matériau à grains plus gros présente une pente d'équilibre naturel (talus au repos) plus raide, en particulier dans le cas des faibles hauteurs, que celle obtenue avec des matériaux à grains fins présentant une courbe granulométrique de même formé.

Ce qu'on vient de dire montre que les résultats obtenus sur modèle et par essais au laboratoire avec des matériaux à grains fins ne doivent pas être directement utilisés ou simplement extrapolés sans envisager d'autres facteurs influençant les propriétés mécaniques des matériaux à gros grains.

\section{Facteurs déterminant les propriétés mécani- ques des matériaux à gros grains.}

La caractéristique principale des matériaux à gros grains est de comporter un grand nombre de grains de grandes dimensions. Ceci impose une étude approfondie de l'importance qu'exercent la grandeur, la forme, l'état de surface, la résistance et les rapports entre les grains. Nous nous bornerons aux paramètres les plus importants très souvent utilisés par lesquels on tente d'habitude d'atteindre la "similitude" des conditions des essais et du comportement du matériau dans sa situation réelle.

\subsection{La similitude géométrique de la composition granulométrique}

C'est une notion appliquée pour la formation d'un matériau de grosseur réduite. Le diamètre nominal des grains du matériau réel dont on forme l'échantillon pour l'essai est diminué d'un rapport constant A :

$$
\mathrm{A}=\frac{\mathrm{D}_{\max }^{r}}{\mathrm{D}_{\max }^{\mathrm{e}}}=\frac{\mathrm{D}_{75}^{r}}{\mathrm{D}_{75}^{\mathrm{e}}}=\frac{\mathrm{D}_{50}^{r}}{\mathrm{D}_{50}^{\mathrm{e}}}=\ldots=\frac{\mathrm{D}_{i}^{r}}{\mathrm{D}_{i}^{\mathrm{e}}} \text {. }
$$

$\mathrm{D}^{r}=$ dimension des grains du matériau réel.

$\mathrm{D}^{\mathrm{e}}=$ dimension des grains de l'échantillon.

Toutes ces courbes peuvent être représentées sur un diagramme granulométrique sous forme adimensionnelle par une courbe exprimée en fonction du logarithme de

$$
\frac{D_{i}^{r}}{D_{50}^{r}}=\frac{D_{i}^{e}}{D_{50}^{e}}=\frac{D_{i}}{D_{0}} \text { ou } \frac{D_{i}^{r}}{D_{\max }^{r}}=\frac{D_{i}^{e}}{D_{\max }^{e}}=\frac{D_{i}}{D_{0}} \text {. }
$$

$D_{i}=$ diamètre nominal du grain défini par le côté de l'ouverture du tamis laissant passer $i \%$ du poids du matériau.

$D_{0}=$ diamètre nominal de référence.

Étant donné que la forme des courbes exprimées en fonction de $D_{1} / D_{50}$ et $D_{1} / D_{\max }$ coïncide avec la forme des courbes exprimée en fonction de $D_{i}^{r}$ et $D_{i}^{e}$, les recherches peuvent être poursuivies sur n'importe laquelle de ces courbes.

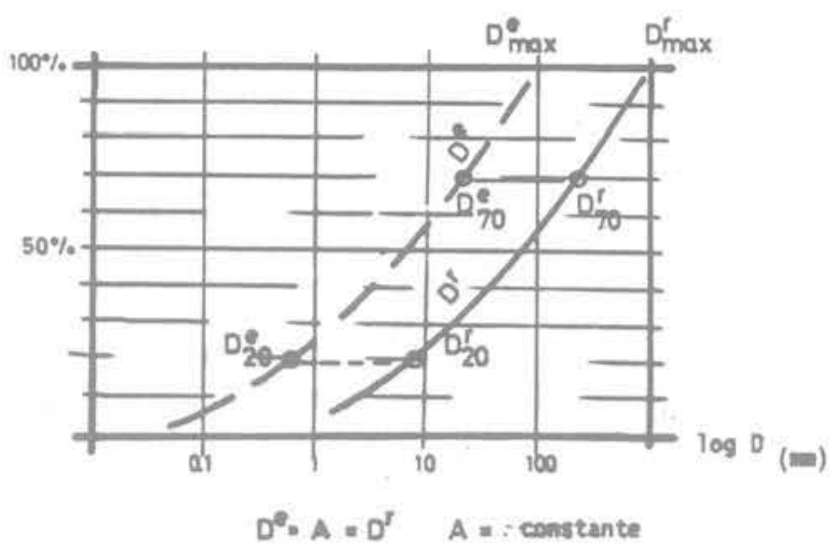

- echantilion $\quad r=$ matériau rél

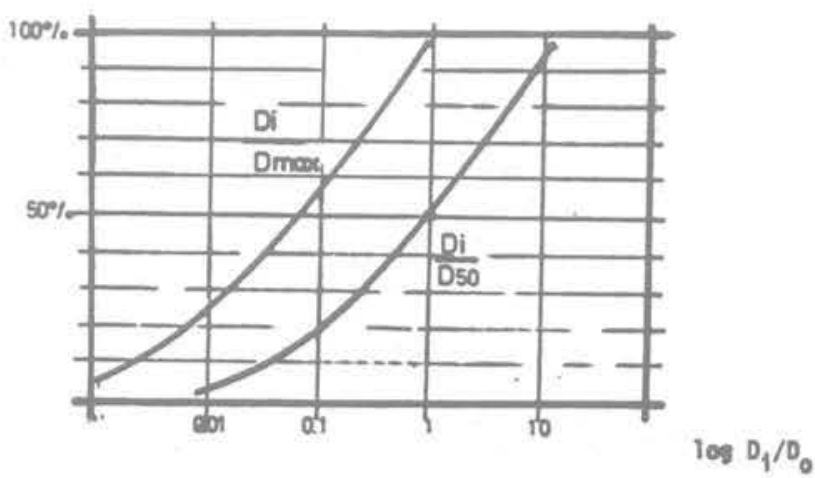

Figure 1 


\subsection{La similitude de porosité}

C'est aussi une notion utilisée pour la formation des matériaux pour les essais ou modèles. La porosité dépend du mode de répartition des grains. Pour les grains ayant à peu près une même forme sphérique on peut déterminer d'après un examen géométrique, en fonction du nombre de points de contact, que la porosité $n$ varie entre $n_{\min }=25 \%$ et $n_{\max }=50 \%$ $\left(n=V_{p} / V_{t}\right.$ rapport du volume des pores $V_{p}$ au volume total $V_{t}$ ), valeurs correspondant aux arrangements spaciaux quadratique ou hexagonal. II y a donc entre 25 et $50 \%$ d'espace libre entre les gros grains pour abriter les petits grains.

On désigne par $D_{i}$ le diamètre des grains uniformes pouvant s'inscrire dans les vides entre les grains uniformes de diamètre $D_{i+1}$. On obtient alors la relation :

$$
(1+n) D_{i}^{3}<n D_{i+1}^{3}
$$

Les courbes limites satisfaisant cette condition sont :

$$
\begin{array}{ll}
1,25 D_{i}^{3}<0,25 D_{i+1}^{3} & \text { soit } \quad \frac{D_{i+1}^{3}}{D_{i}^{3}}>5=\lambda \\
1,50 D_{i}^{3}<0,50 D_{i+1}^{3} & \text { soit } \quad \frac{D_{i+1}^{3}}{D_{i}^{3}}>3=\lambda .
\end{array}
$$

Ces expressions ont été établies en supposant que le volume total rempli par les grains de diamètre plus petit est égal au volume des pores qui existe entre les grains de diamètre plus grand à savoir :

$$
\begin{gathered}
V_{p}^{i+1}=V_{t}^{i}=V_{g}^{i}+V_{p}^{i} \\
\alpha D_{i+1}^{3} \cdot n>\beta D_{i}^{3}+\alpha D_{i}^{3} \cdot n
\end{gathered}
$$

où l'on suppose que les coefficients $\beta$ et $\alpha$ sont égaux bien qu'en réalité $\beta$ soit moins élevé que $\alpha$.

Avec les notations suivantes:

$\mathrm{V}_{\mathrm{p}}^{\mathrm{i}+1}=$ volume des vides ou pores entre les grains de la fraction $(i+1)$;

$V_{t}^{i}=$ volume total qui est égal à la somme de $\mathrm{V}_{\mathrm{p}}^{\mathrm{i}}=$ volume des vides et $\mathrm{V}_{\mathrm{o}}^{\prime}=$ volume du grain de la fraction (i).
Dans ce cas on obtient :

$$
\begin{aligned}
\alpha D^{3} \cdot n & =V_{p} \\
\beta D^{3} & =V_{g}
\end{aligned}
$$

où $\alpha$ et $\beta$ sont des coefficients et $n$ représente la porosité.

La figure 2 présente les courbes limites pour $n=25 \%$ et $n=50 \%$ définissant les conditions de remplissage des pores des gros grains par les petits grains.

Les courbes limites de la figure 2 indiquent que ces conditions théoriques ne sont satisfaites que par une petite bande de courbes granulométriques. Pour la plupart des matériaux réels la répartition des grains dans la masse ne suit que partiellement cette image idéale et en majeure partie les grains d'une même dimension sont séparés c'est-à-dire sans contact entre eux mais en contact aussi bien avec des grains plus gros qu'avec des grains plus petits.

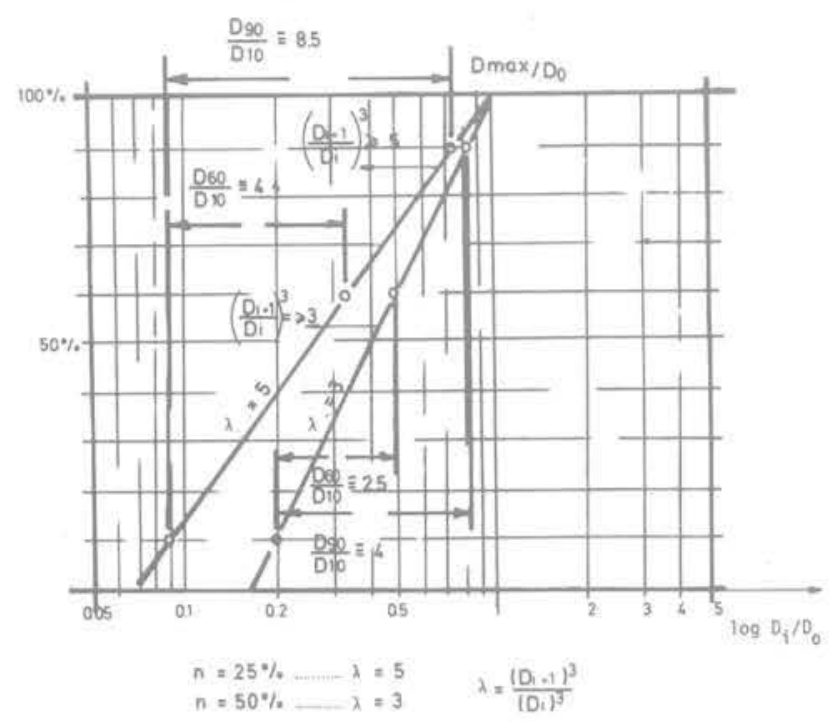

Figure 2

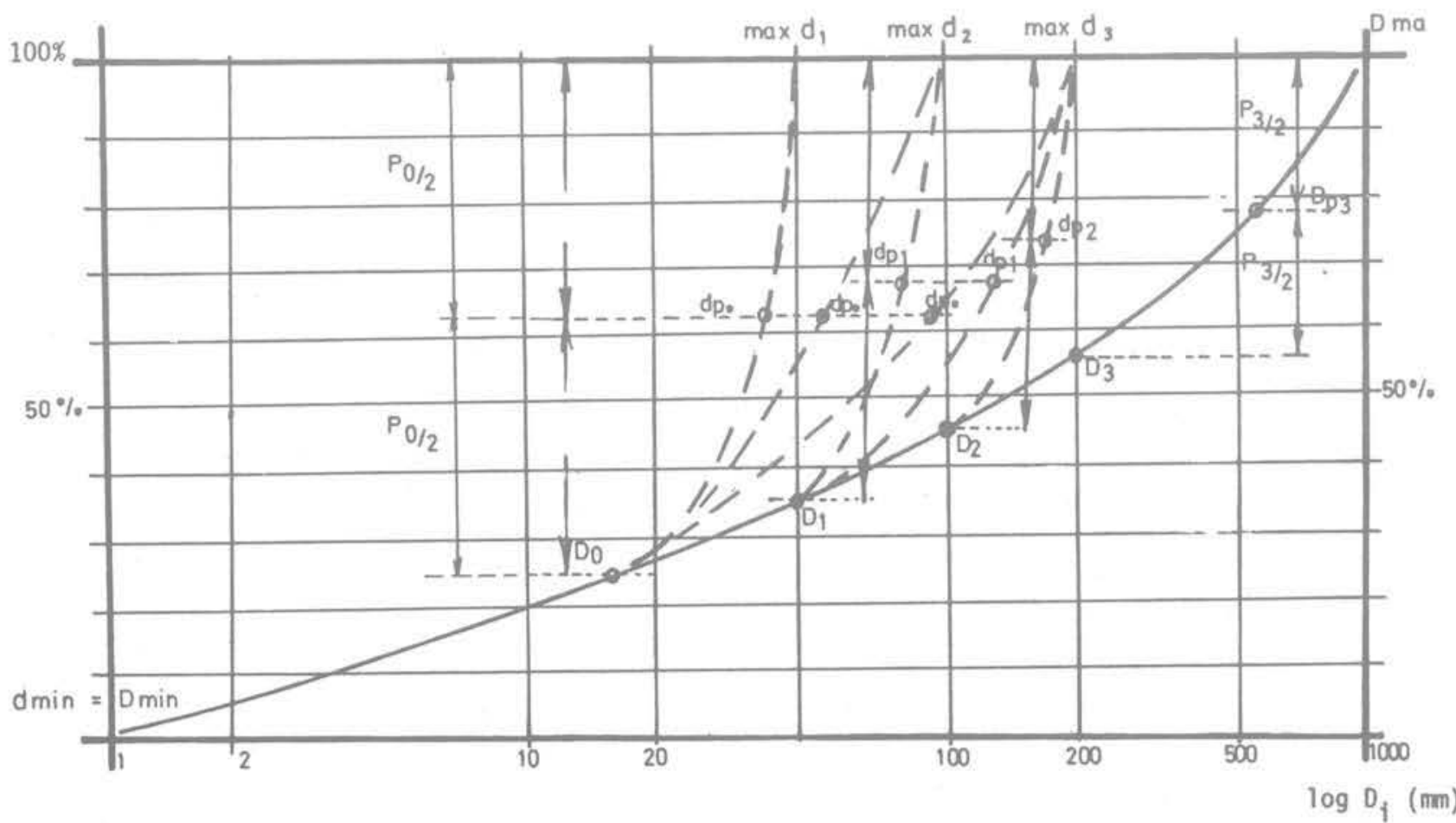

Figure 3 
On a tenté (Frost 1972) d'obtenir pour la courbe granulométrique de l'échantillon la similitude en grandeur des forces aux contacts des grains, ou bien de maintenir dans le volume représentatif le même nombre de grains. Un tel abandon de la conception de la similitude géométrique conduit à des interprétations plus compliquées qui se traduisent tout de même par la détermination expérimentale de la dépendance de certains paramètres géométriques et des propriétés de déformabilité ou de résistance au cisaillement.

La figure 3 présente un exemple de modélisation sans similitude géométrique où l'on "agrandit " progressivement le grain moyen représentatif des granulométries de l'échantillon dans les intervalles:

$$
\begin{array}{lll}
D_{0}-\max d_{1} ; & D_{1}-\max d_{2} ; & D_{2}-\max d_{3} ; \\
D_{0}-\max d_{2} ; & D_{1}-\max d_{3} ; \\
D_{0}-\max d_{3} . &
\end{array}
$$

D'après les variations de mesures de densité et de déformabilité, on établit une corrélation valable pour les intervalles:

$$
D_{0}-D_{\max } ; D_{1}-D_{\max } ; D_{2}-D_{\max } \text {. }
$$

La similitude de porosité doit être par conséquent harmonisée avec la similitude géométrique de la composition granulométrique sinon on aboutit à la comparaison du comportement de matériaux de structure différente. Si l'on arrive à réaliser la similitude géométrique de la composition granulométrique (et de la forme des grains), la porosité équivalente de l'échantillon est alors identique.

\subsection{La similitude du volume représentatif}

Pour le matériau à gros grains le comportement mécanique est observé sur un volume représentatif de ce matériau. Ce volume représentatif doit être assez grand pour permettre le déroulement de tous les processus et de toutes les interactions entre les grains sans facteurs limitatifs et sans qu'il y ait d'influences. imposées par les dimensions des modèles ou des échantillons. Ces interactions sont réduites aux:

- déplacements relatifs des grains par glissement et roulement.

- déformations élastiques de contact,

- destructions des grains par fragmentation avec modification de la composition granulométrique.

Chacune de ces interactions est liée à la dissipation de la plus grande part de l'énergie liée à la déformation irréversible et à la conservation d'une partie moindre de l'énergie liée à la déformation élastique du grain.

La détermination directe du volume représentatif par l'observation du comportement du grain est très compliquée; toutefois, pour chaque type d'essai ou de modèle on peut déterminer une dimension minimale d'échantillon au-delà de laquelle une augmentation des dimensions ne donne pas de différence pouvant être mesurée pour les paramètres de résistance ou de déformabilité.

\subsection{La similitude de la qualité du grain}

Les paramètres de similitude doivent être déterminés pour les propriétés suivantes du grain :

- déformabilité du grain.

- résistance à la fragmentation, c'est-à-dire par cassure fragile,

- résistance au déplacement relatif grain sur grain par translation et rotation (en fonction de la rugosité de la surface, de la forme et du mode d'assemblage des grains, c'est-à-dire de la porosité).

Pour les matériaux réels, on ne peut pas réaliser une similitude particulière de la qualité du grain. Pour des billes de verre, d'acier ou des matériaux semblables ceci peut être effectué plus facilement, bien qu'il y ait des problèmes de similitude en grandeur des forces apparaissant aux contacts, ce qui implique également des problèmes d'équivalence des contraintes moyennes. La même contrainte moyenne dans un plan d'intersection du volume représentatif se traduit par des forces de contact considérablement plus élevées, ce qui fausse la similitude du comportement aussi bien du point de vue de la déformabilité, qu'en ce qui concerne la résistance. Les forces de contact plus élevées provoquent la cassure des gros grains (présentant un plus grand nombre de fissures que les petits grains, de sorte qu'ils sont en général plus sensibles à la cassure fragile). Simultanément la résistance au cisaillement diminue sous l'effet des concentrations plus élevées des contraintes normales aux contacts des grains de sorte que la consommation de l'énergie de déformation se répartit inégalement entre les matériaux de grosseur plus élevée et ceux ayant des grains moins grands.

Étant donné les. difficultés rencontrées pour la détermination de l'énergie dépensée dans chacun des aspects mentionnés de la déformation du grain, on s'efforce de constater la similitude du comportement global et notamment pour le changement du volume représentatif (dilatance) et pour une composition granulométrique qui ne soit pas modifiée lors du cisaillement à volume constant par rapport à la composition granulométrique initiale, c'est-à-dire dans une phase où l'on a atteint la porosité critique correspondant à l'état limite de l'équilibre à volume constant.

Ces deux conditions peuvent être réalisées par des essais sur un matériau dont la composition pétrographique, la forme des grains, la porosité initiale et la forme de la courbe granulométrique sont semblables.

Avec les conditions de similitude ainsi définies, on peut examiner l'influence de la dépense d'énergie pour la fragmentation du grain en fonction de la contrainte moyenne existant sur l'échantillon. Cette relation a un caractère empirique spécifique pour chaque type de matériau.

\section{Analyse des résultats des essais de résis- tance au cisaillement}

L'analyse globale du comportement du matériau granulaire lors de la déformation de glissement dans des conditions de déformation plane peut être effectuée en observant le volume représentatif sur lequel agissent les forces verticale normale $\mathrm{V}$ et horizontale $\mathrm{H}$ de cisaillement. Pour des matériaux très compacts il se produit une dilatance. Les déplacements relatifs se produisent avec un certain angle par rapport à la direction de la force $\mathrm{H}$, et la déformation du volume représentatif peut s'exprimer par:

$$
\check{\delta}=\vec{\delta}_{x}+\vec{\delta}_{y}
$$

valeurs vectorielles présentées sur la figure 4 . 


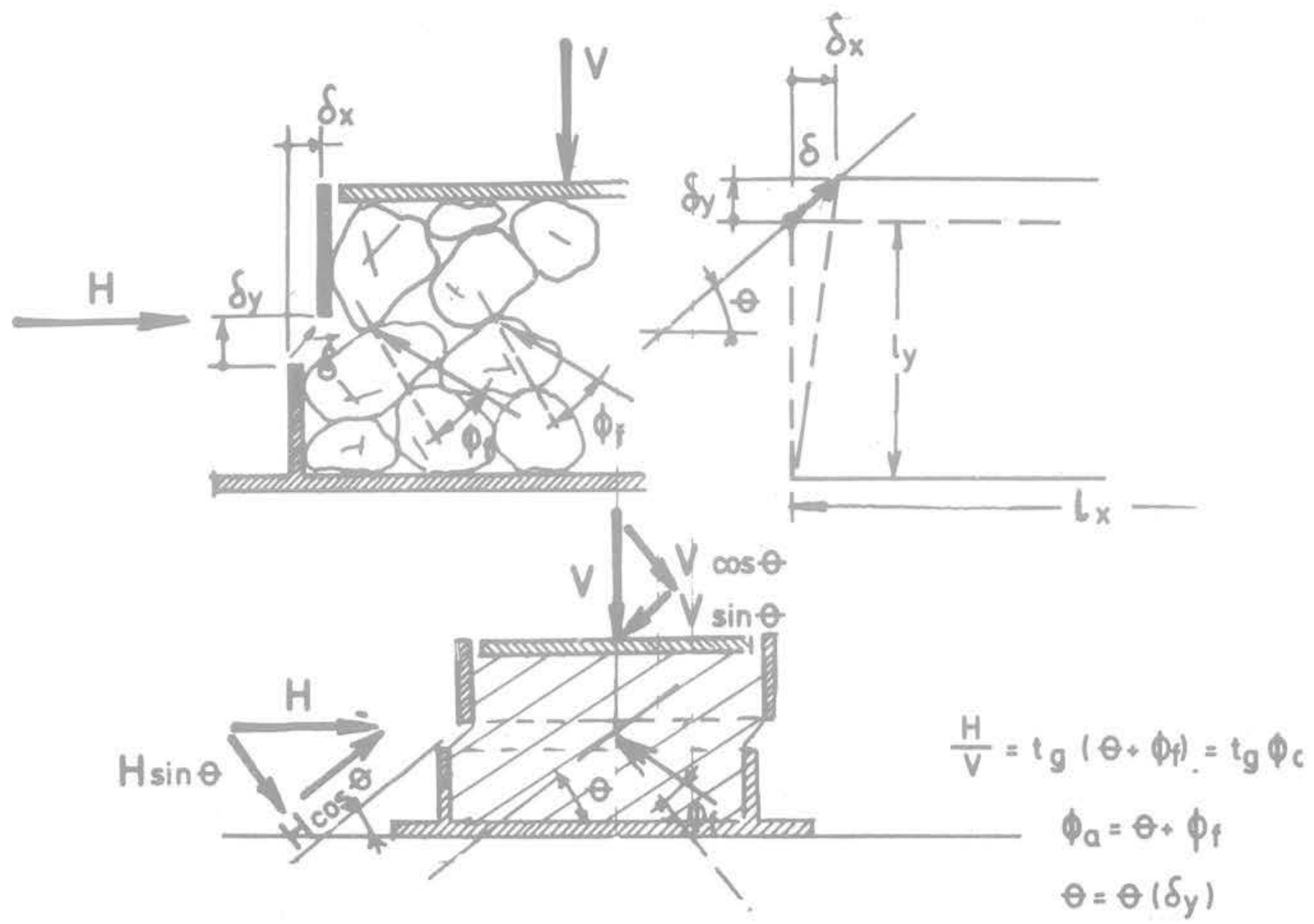

Figure 4

$$
\frac{\delta_{y}}{\delta_{x}}=\operatorname{tg} \theta ; \quad \frac{\delta_{x}}{\ell_{y}+\ell_{x}} \# \frac{\delta_{y}}{\ell_{y}}=\delta y \text { glissement. }
$$

Changement de volume :

$$
\begin{aligned}
\delta V & =\frac{V_{0}-V_{1}}{V_{0}}=\frac{\ell_{x} \cdot \ell_{y}-\ell_{x}\left(\ell_{y}+\delta_{y}\right)}{\ell_{x} \cdot \ell_{y}} \\
& =-\frac{\delta_{y}}{\ell_{y}}=-\frac{\delta_{y}}{\delta_{x}} \cdot \delta y
\end{aligned}
$$

$$
\text { d'oủ } \quad \operatorname{tg} \theta=\frac{\delta_{y}}{\delta_{x}}=-\frac{\delta V}{\delta y}
$$

(+ $\delta \mathrm{V}$ compression; $-\delta \mathrm{V}$ dilatance).

Partant des conditions d'équilibre dans le sens du déplacement $\delta$ nous obtenons d'après la figure 4 :

$H \cdot \cos \theta=V \cdot \sin \theta+H \cdot \sin \theta \cdot \operatorname{tg} \phi_{t}+V \cdot \cos \theta \cdot \operatorname{tg} \phi_{t}$ d'où : $H=V \cdot \operatorname{tg} \theta+H \cdot \operatorname{tg} \theta \cdot \operatorname{tg} \phi_{1}+V \cdot \operatorname{tg} \phi_{t}$.

$V \cdot \operatorname{tg} \theta$ dépend de $\delta_{y} / \delta_{x} ;$ pour $\delta_{y}=0, V \cdot \operatorname{tg} \theta=0$.

$V \cdot \operatorname{tg} \phi_{t}$ dépend uniquement du frottement réel, pour $\delta_{y}=0$ comme pour $\delta_{y} \neq 0$.

$H \cdot \operatorname{tg} \theta \cdot \operatorname{tg} \theta_{t}:$ dépense additionnelle d'énergie, proportionnelle à la force $\mathrm{H}$.

On en déduit :

$$
\begin{aligned}
& H=V\left(\frac{\operatorname{tg} \theta}{1-\operatorname{tg} \theta \cdot \operatorname{tg} \phi_{f}}+\frac{\operatorname{tg} \phi_{t}}{1-\operatorname{tg} \theta \cdot \operatorname{tg} \phi_{t}}\right) \\
& H=V \frac{\operatorname{tg} \theta+\operatorname{tg} \phi_{t}}{1-\operatorname{tg} \theta \cdot \operatorname{tg} \phi_{f}}=V \cdot \operatorname{tg}\left(\theta+\phi_{t}\right) .
\end{aligned}
$$

La résistance moyenne ou apparente peut être exprimée par :

$$
\frac{\mathrm{H}}{\mathrm{V}}=\operatorname{tg}\left(\theta+\phi_{\mathrm{t}}\right)=\operatorname{tg} \phi_{\mathrm{a}}
$$

en posant: $\quad \phi_{\mathrm{a}}=\theta+\phi_{\mathrm{f}}$

L'angle de frottement $\phi_{t}$ est soit constant, soit une fonction de $\sigma_{\text {oct, }}$ c'est-à-dire de la contrainte normale sphérique, et $\theta$ dépend de $\sigma_{\text {oct }}$ et $\tau_{\text {oct }}$ de la valeur du changement de volume et de la cassure du grain. Les essais de cisaillement direct ( $\mathrm{V}=$ const.) peuvent donner les paramètres $\theta$ et $\phi_{t}$ si les appareils sont suffisamment adaptés aux grands déplacements et si I'on a mesuré les déplacements verticaux.

Dans l'essai standard de compression triaxiale pour le matériau isotrope, on obtient l'expression analogue :

$$
\begin{gathered}
\frac{\sigma_{1}}{\sigma_{3}}=\operatorname{tg}^{2}\left(45+\frac{\phi_{a}}{2}\right) ; \\
\frac{\sigma_{1}-\sigma_{3}}{\sigma_{1}+\sigma_{3}}=\sin \phi_{\mathrm{a}} \text { où } \phi_{\mathrm{a}}=\theta+\phi_{\mathrm{t}} .
\end{gathered}
$$

Pour $\theta=0$ on obtient $\frac{\sigma_{1 \mathrm{cv}}}{\sigma_{3}}=\operatorname{tg}^{2}\left(45+\frac{\phi_{t}}{2}\right)$ représentant le cas où la fragmentation s'effectue sans changement de volume.

L'énergie totale (travail) $\delta E$ dépensée pour la déformation de l'échantillon s'écrit :

$$
\delta \mathrm{E}=\delta \mathrm{U}+\delta \mathrm{W}
$$




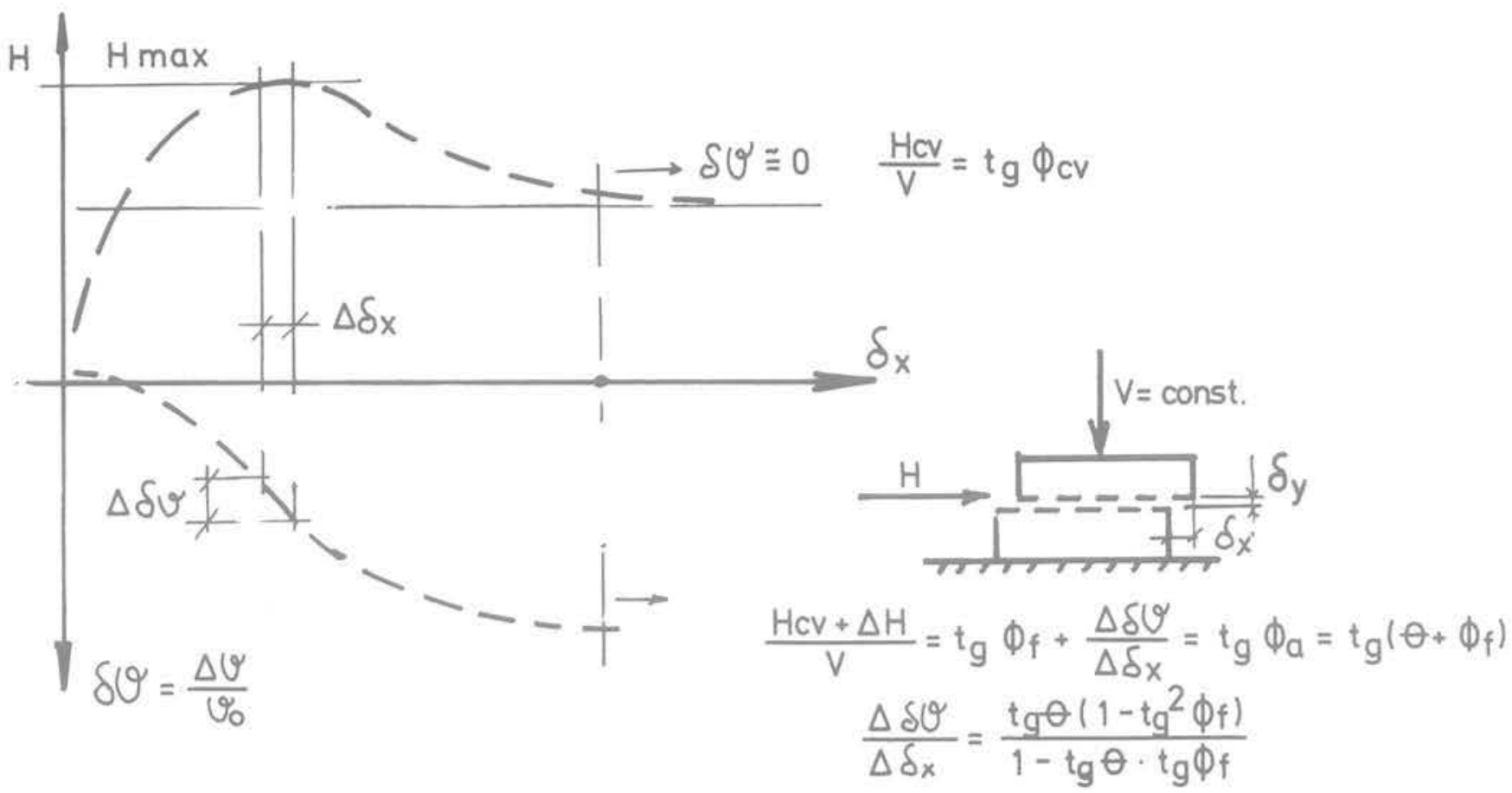

Figure 5

où : $\delta \mathrm{U}=$ travail emmagasiné dû à la déformation élastique de l'échantillon :

$$
\begin{gathered}
\delta U=p \cdot \delta V_{e} \text { où } p=\frac{1}{3}\left(\sigma_{1}+2 \cdot \sigma_{3}\right) \\
\delta V_{e}=\delta \varepsilon_{1 e}+2 \cdot \delta \varepsilon_{3 e} .
\end{gathered}
$$

$\delta \mathrm{W}=$ travail dépensé irréversiblement dû à la déformation non élastique de l'échantillon:

$$
\delta \mathrm{W}=\sigma_{1} \cdot \delta \varepsilon_{1 \mathrm{~s}}+\sigma_{3} \cdot 2 \cdot \delta \varepsilon_{3 \mathrm{~s}} .
$$

Étant donné que :

$$
\delta \mathrm{V}_{\mathrm{s}}=\delta \varepsilon_{1 \mathrm{~s}}+2 \cdot \delta \varepsilon_{3 \mathrm{~s}}
$$

On obtient :

$$
\begin{aligned}
& \delta \mathrm{W}=\sigma_{1} \cdot \delta \varepsilon_{1 \mathrm{~s}}+2 \cdot \sigma_{3} \cdot \frac{1}{2}\left(\delta \mathrm{V}_{\mathrm{s}}-\delta \varepsilon_{1 \mathrm{~s}}\right) \\
& \delta \mathrm{W}=\left(\sigma_{1}-\sigma_{3}\right) \cdot \delta \varepsilon_{1 \mathrm{~s}}+\sigma_{3} \cdot \delta \mathrm{V}_{\mathrm{s}} .
\end{aligned}
$$$$
\text { D'où : } \quad \frac{\delta \mathrm{W}}{\delta \varepsilon_{1 \mathrm{~S}}}=\left(\sigma_{1}-\sigma_{3}\right)+\sigma_{3} \frac{\delta \mathrm{V}_{\mathrm{S}}}{\delta \varepsilon_{1 \mathrm{~S}}} \text {. }
$$

C'est-à-dire pour $\delta V_{S}=0$ :

$$
\frac{\delta \mathrm{W}}{\delta \varepsilon_{1 \mathrm{~s}}}=\left(\sigma_{1}-\sigma_{3}\right)_{\mathrm{cV}}=\left(\sigma_{1}+\sigma_{3}\right) \cdot \sin \phi_{4} .
$$

De façon analogue on obtient:

$$
\begin{gathered}
\left(\sigma_{1}+\sigma_{3}\right) \cdot \sin \phi_{\mathrm{a}}=\left(\sigma_{1}-\sigma_{3}\right)_{\mathrm{cv}}+\Delta\left(\sigma_{1}-\sigma_{3}\right) \\
\text { avec : } \quad \Delta\left(\sigma_{1}-\sigma_{3}\right)=\sigma_{3} \frac{\delta \mathrm{V}_{\mathrm{S}} .}{\delta \varepsilon_{1 \mathrm{~s}}}
\end{gathered}
$$

On obtient ainsi :

$$
\sin \phi_{\mathrm{a}}=\sin \phi_{\mathrm{f}}+\frac{\sigma_{3}}{\sigma_{1}+\sigma_{3}} \cdot \frac{\delta \mathrm{V}_{\mathrm{s}}}{\delta \varepsilon_{1 \mathrm{~s}}} .
$$

Cette relation peut être représentée sur le cercle de Mohr par :

$$
\pm \sigma_{3} \frac{\delta V_{S}}{\delta \varepsilon_{1 S}}= \pm \Delta\left(\sigma_{1}-\sigma_{3}\right) \text { quand } \sigma_{1}+\sigma_{3}=\text { const. }
$$

déterminant la valeur $\sigma_{3}$ par rapport à la valeur $\sigma_{3 c}$ correspondant à

$$
\frac{\delta V_{S}}{\delta \varepsilon_{1 S}}=0
$$

P. Rowe (1962) interprète l'expression :

$$
\frac{\delta W}{\delta \varepsilon_{1 s}}=\left(\sigma_{1}-\sigma_{3}\right)+\sigma_{3} \frac{\delta V_{S}}{\delta \varepsilon_{1 s}}
$$

en définissant :

$$
\frac{\delta \mathrm{W}}{\delta \varepsilon_{1 \mathrm{~s}}}=\left(\sigma_{1}-\sigma_{3}\right)_{\mathrm{a}} \text { pour } \quad \frac{\delta \mathrm{V}_{\mathrm{S}}}{\delta \varepsilon_{1 \mathrm{~s}}} \neq 0
$$$$
\text { d'où : } \quad\left(\sigma_{1}-\sigma_{3}\right)_{\mathrm{a}}=\sigma_{1}-\sigma_{3}\left(1-\frac{\delta \mathrm{V}_{\mathrm{s}}}{\delta \varepsilon_{1 \mathrm{~s}}}\right) \text {. }
$$

A. Bishop (1965) interprète la même expression sous la forme :

et

$$
\begin{array}{lll}
\frac{\delta \mathrm{W}}{\delta \varepsilon_{1 \mathrm{~s}}}=\sigma_{1 \max }-\sigma_{3} & \text { pour } & \frac{\delta V_{\mathrm{S}}}{\delta \varepsilon_{1 \mathrm{~s}}} \neq 0 \\
\frac{\delta \mathrm{W}}{\delta \varepsilon_{1 \mathrm{~S}}}=\sigma_{1 \mathrm{CV}}-\sigma_{3} & \text { pour } & \frac{\delta V_{\mathrm{S}}}{\delta \varepsilon_{1 \mathrm{~S}}}=0
\end{array}
$$

d'où : $\quad \sigma_{1 \text { max }}-\sigma_{3}=\sigma_{1 C V}-\sigma_{3}+\sigma_{3} \frac{\delta V_{S}}{\delta \varepsilon_{1 S}}$

on en déduit :

$$
\sigma_{1 \max }-\sigma_{1 \mathrm{CV}}=\sigma_{3} \frac{\delta \mathrm{V}_{\mathrm{s}}}{\delta \varepsilon_{1 \mathrm{~s}}}
$$

déterminant la correction de la valeur $\sigma_{1}$ par rapport à $\sigma_{1 \mathrm{cv}}$ correspondant à

$$
\frac{\delta V_{s}}{\delta \varepsilon_{1 s}}=0
$$

Ces deux interprétations permettent de considérer que le travail additionnel dépend de la force additionnelle de résistance de l'échantillon dont l'action complète est imputée à la contrainte $\sigma_{3}$ (P. Rowe), ou bien que le travail additionnel dépend de la force active additionnelle qu'on impute intégralement à la contrainte $\sigma_{1}$ (A. Bishop), ce qui peut être représenté sur les cercles de Mohr (figure 7). 

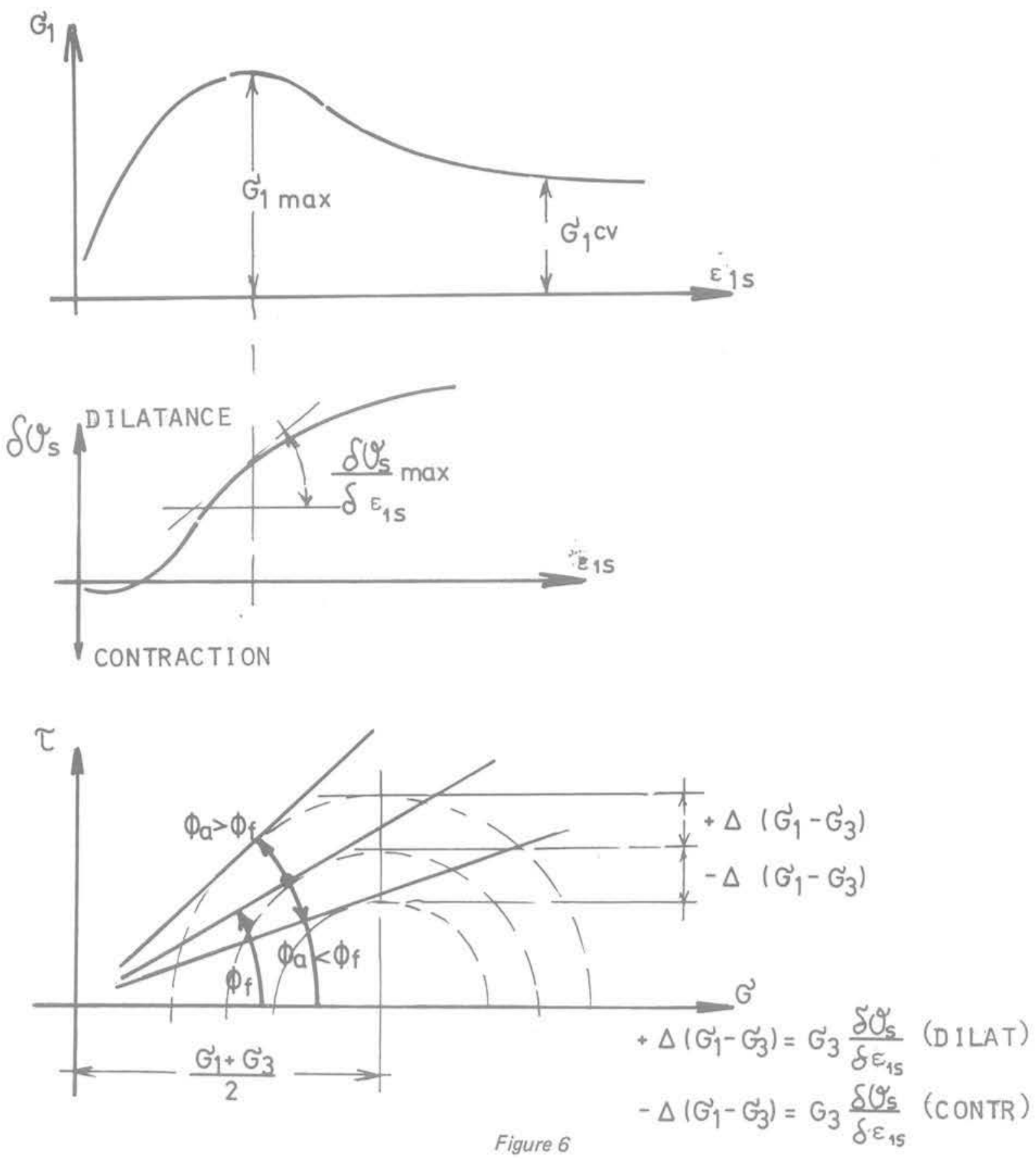

(A. BISHOP)

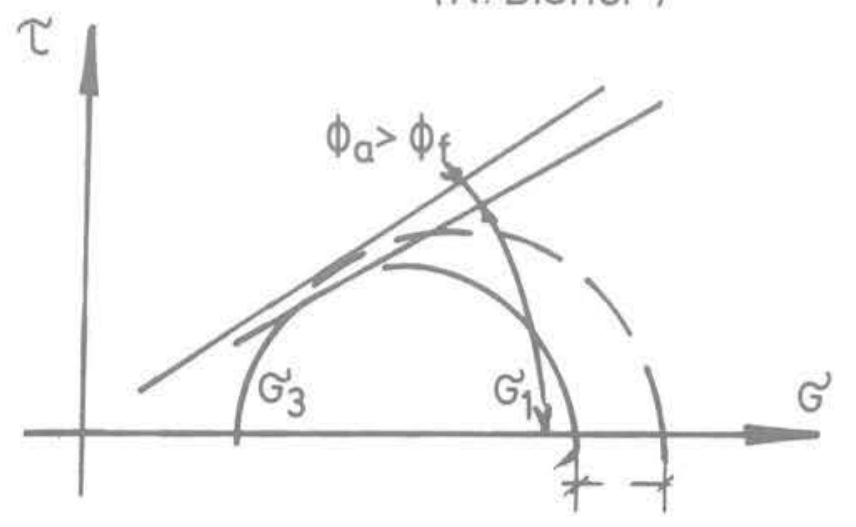

$\Delta G_{1}=\frac{\delta U_{S}}{\delta E_{1 S}} * G_{3}$

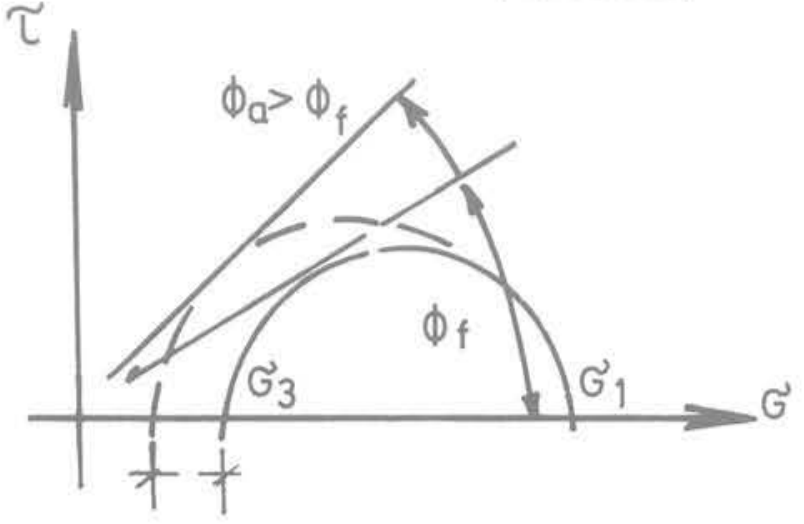

$$
\Delta G_{3}=\frac{\delta V_{s}}{\delta \text { IS }} \times G_{3}
$$

Figure 7 
On a nettement démontré dans l'interprétation initiale que $\phi_{a}$ est considérablement plus élevé que $\phi_{t}$ si $\delta V_{S}$ est une dilatance et inversement.

Étant donné que lors de l'essai de cisaillement direct le changement de volume se répercute sur le travail additionnel effectué par les forces normale $(\mathrm{V} \cdot \operatorname{tg} \phi)$ et de cisaillement $\left(\mathrm{H} \cdot \operatorname{tg} \theta \cdot \operatorname{tg} \phi_{t}\right)$, l'interprétation la plus adéquate est celle définissant :

$$
\frac{\delta V_{s}}{\delta \varepsilon_{1}}=\frac{\Delta\left(\sigma_{1}-\sigma_{3}\right)}{\sigma_{3}}
$$

qui a été représentée sur la figure 6 .

Pour une analyse semblable on utilise souvent le rapport entre $\sigma_{1}$ et $\sigma_{3}$

$$
\frac{\sigma_{1}}{\sigma_{3}}=R_{\sigma}=D \cdot K
$$

où :

$\mathrm{D}=1$ pour $\delta \mathrm{V}=0$ et $\mathrm{D}>1$ pour une dilatance $\delta \mathrm{V}<0$,

$D<1$ pour une contraction $\delta V>0$;

$\mathrm{K}$ est le coefficient proportionnel à la résistance maximale de l'échantillon.

$$
K=\operatorname{tg}^{2}\left(45+\frac{\phi_{t}}{2}\right) \quad \text { (P. Rowe, 1963). }
$$

Avec $\delta \varepsilon_{1}>0$ on peut écrire: $D=\left(1-\frac{\delta V}{\delta \varepsilon_{1}}\right)$ et l'on a :

$$
\frac{\sigma_{1}}{\sigma_{3}}=R=\operatorname{tg} \alpha \cdot \operatorname{tg}\left(\phi_{f}+\theta\right) \quad \text { (P. Rowe, 1963) }
$$

où sont introduits les paramètres $\operatorname{tg} \alpha$ et $\theta$ par lesquels la résistance totale au cisaillement peut être représentée en fonction de l'interaction des grains et des changements de volume de l'échantillon d'une manière plus souple et plus appropriée. Le paramètre $\operatorname{tg} \alpha$ peut être déterminé pour les conditions pour lesquelles la fragmentation du grain ne se produit pas et le paramètre $\theta$ pour les conditions pour lesquelles l'on a la fragmentation du grain lors du changement de volume équivalent.

Toutes les expressions précédentes prennent en considération d'une certaine manière l'existence de la différence de résistance au cisaillement par rapport à l'état sans changement de volume, à savoir au moment où les déplacements relatifs du grain s'effectuent avec la contrainte normale permanente et la contrainte de cisaillement, c'est-à-dire quand le travail entier est dépensé pour maîtriser les résistances alors que ni la composition granulométrique ni le volume des pores dans l'échantillon ne se modifient.

En même temps ces expressions offrent la possibilité de déterminer la relation entre les coefficients $\phi_{\text {, et }} \theta$ ou $\phi_{i}, \theta$ et $\alpha$ vis-à-vis des facteurs suivants :

a) qualité de la surface du grain,

b) porosité et changement de volume,

c) influence de la destruction du grain.

Pour les matériaux ayant une composition granulométrique et une qualité de surface des grains semblables, on peut constater une relation du type :

$$
\phi=\phi_{0}-\frac{\bar{\sigma}_{i}}{A+B \bar{\sigma}_{i}}
$$

où $\phi=$ angle de la tangente au cercle de Mohr, $\phi_{0}=$ angle de la tangente pour $\sigma_{1}=0$,

$\bar{\sigma}_{i}=$ contrainte caractéristique ou invariance dans les conditions limites de l'échantillon (M. Maksimovic, 1978).

Si la valeur $\bar{\sigma}_{i}-\bar{\sigma}_{i \max }$ augmente, la valeur $\phi_{0}-\phi_{\text {min }}$ tend vers une valeur constante:

$$
\phi_{0}-\phi_{\min } \longrightarrow \frac{1}{B} \text {. }
$$

En modifiant le degré de compacité de l'échantillon (c'est-à-dire sa porosité) on peut aboutir à $\phi_{0}-\phi_{\min }=$ $1 / B$, avec $V_{s}=0$, c'est-à-dire au cas où le travail entier des forces extérieures s'effectue uniquement pour maîtriser le frottement avec une destruction insignifiante du grain (destruction ne provoquant pas de changement de $V_{s}$ ). Cette valeur de l'asymptote $\phi_{\min }$ et la porosité correspondante $n_{\text {ov }}$ représentent les valeurs limites pour la courbe granulométrique étudiée à savoir $\phi>\phi_{\min }$ et $n<n_{\mathrm{ev}}$ pour toutes les valeurs de $\sigma_{i}$.

En modifiant la courbe granulométrique on peut ensuite déterminer $\phi_{\min }$ et $n_{c v}$ en tant que fonctions de la composition granulométrique, tout en assurant ainsi la possibilité de prévisions des valeurs $\phi_{\min }$ et $\mathrm{n}_{\mathrm{cv}}=\mathrm{n}_{\max }$ pour le matériau réel.

Une telle approche implique l'exécution de quelques séries d'essais à courbe granulométrique semblable et à grosseur différente des grains avec $V_{s}=0 \quad \rho^{+}$ $\left(\phi_{0}-\phi_{\min }\right)=$ const.

Dẻ même que dans l'essai de compressibilité à l'cedomètre, où l'on a une non-homogénéité des forces de contacts entre les grains, la fragmentation est plus accentuée le long des parois de l'appareil, de même dans l'essai de cisaillement direct apparaît une accumulation semblable de forces aux contacts des grains non seulement le long des perois de l'appareil, mais aussi à la surface de la zone de cisaillement. Les déplacements relatif́s des grains dans le sens vertical, provoqués par le déplacement horizontal du cadre supérieur par rapport au cadre inférieur, sont suivis de changement de porosité et de la fragmentation des grains dans cette zone; ces changements s'amortissent à mesure qu'on s'éloigne de la zone du cisaillement. C'est pourquoi, la grandeur des changements réels de volume dans la zone du cisaillement est supérieure à celle mesurée à la surface, et les différences augmentent avec la croissance du rapport de la hauteur de l'échantillon au D maximum du matériau, et avec la diminution de la contrainte verticale, de manière qu'on n'ait pas de forces constantes élevées suivies de la fragmentation et du déplacement relatif considérable du grain atténuant le déplacement vertical de la surface supérieure de l'appareil. C'est pourquoi, la notion du volume représentatif est liée non seulement à l'effet de la paroi métallique entourant les échantillons, mais aussi à la détermination du volume de matériau ayant un comportement homogène (déformations homogènes). Les difficultés qu'on rencontre lors de l'interprétation des résultats du cisaillement direct proviennent en général du fait que cette non-homogénéité de déformation n'est pas prise en considération. 

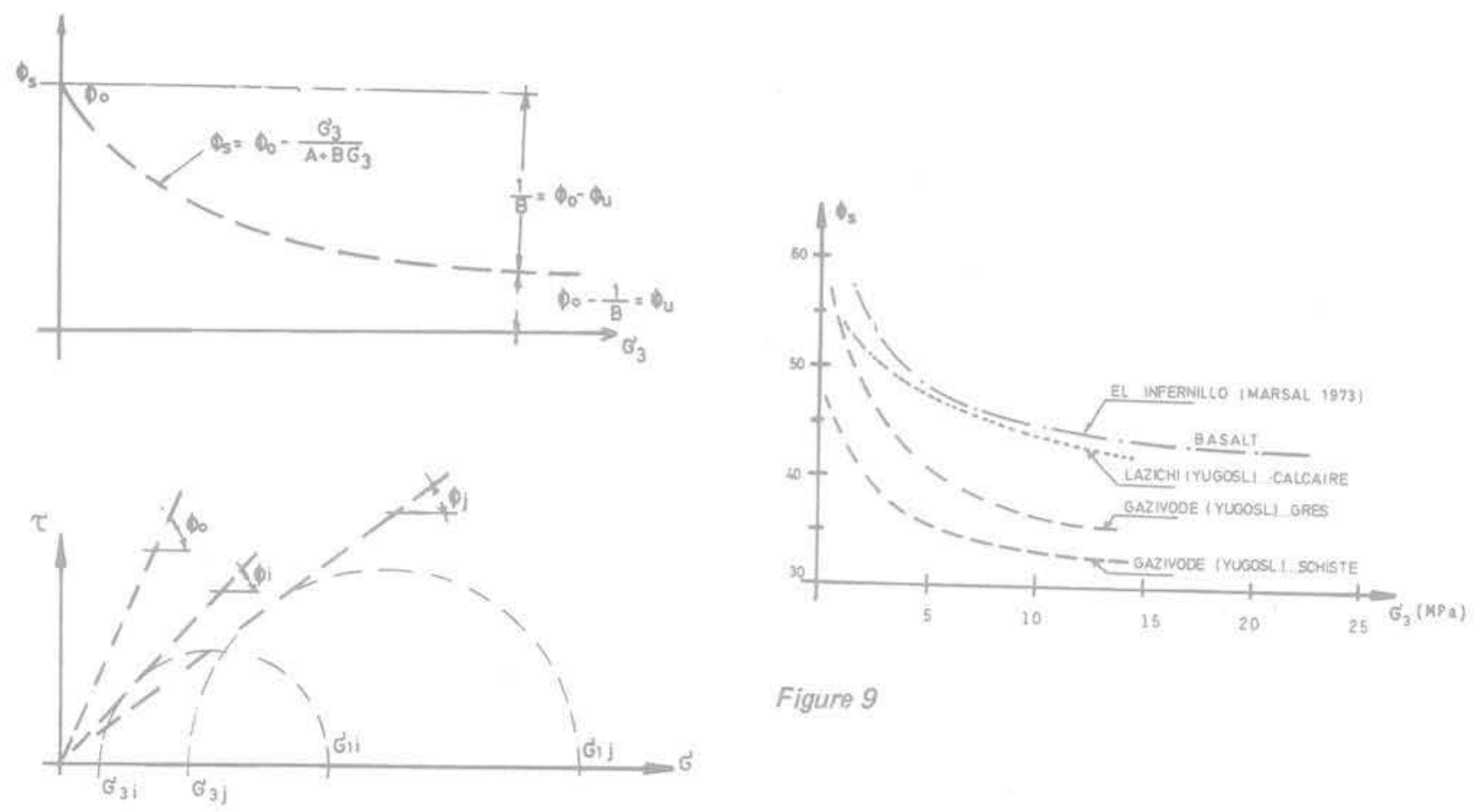

Figure 9

Figure 8

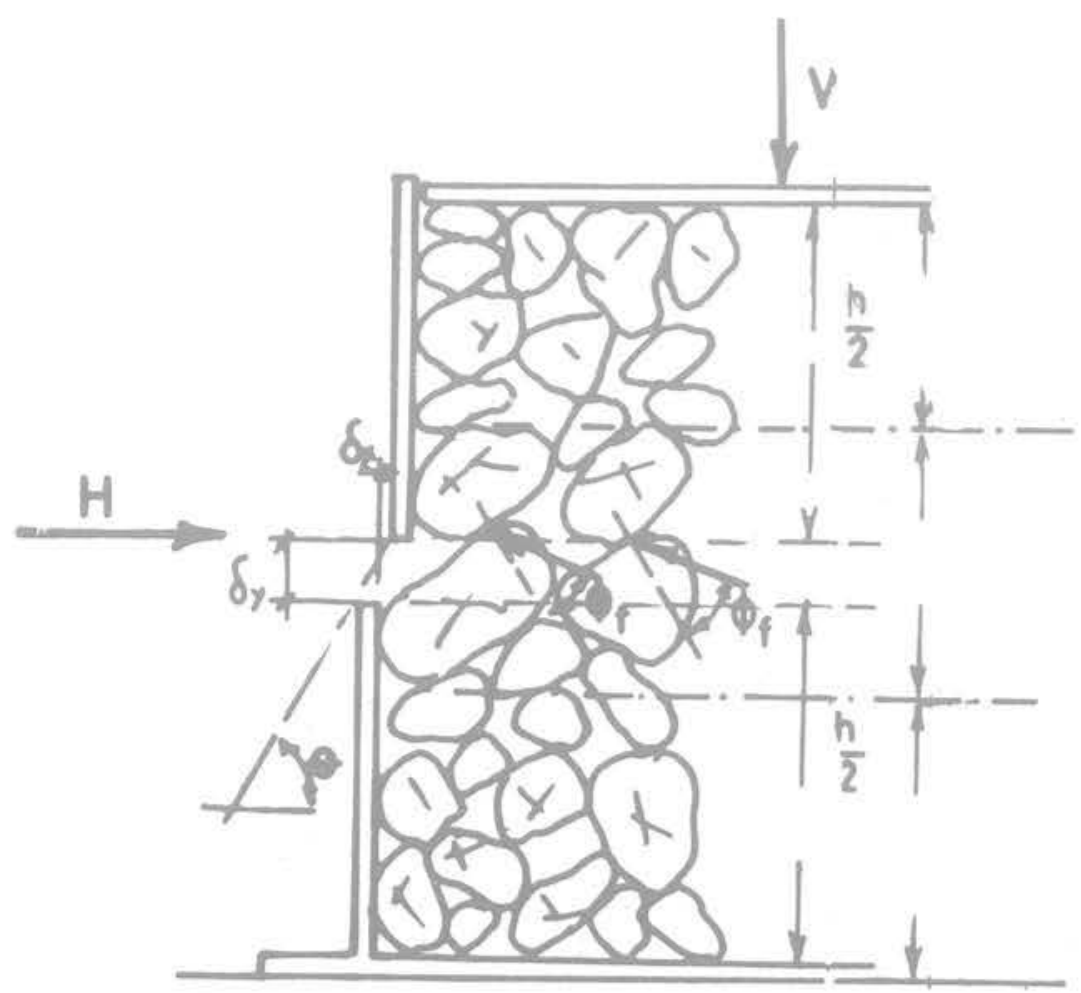

ZONE D'AMORTISSEMENT

DE LA DILATANCE

ZONE DE DILATANCE PAR CISAILLEMENT

ZONE D'AMORTISSEMENT DE LA DILATANCE

Figure 10 


\section{Analyse des résultats des essais de défor- mabilité}

D'une façon générale, la déformabilité du matériau à gros grains dépend des facteurs suivants:

a) déplacement relatif des grains,

b) destruction des grains,

c) déformation élastique des grains.

Les déformations irréversibles a), b), sont considérablement plus élevées que celles réversibles c) et elles participent davantage au changement de la porosité initiale du matériau.

Les déplacements relatifs des grains dépendent de l'état initial de l'assemblage des grains, de leur forme et de leur porosité; ils apparaissent déjà pour des contraintes de faible intensité. La destruction des grains suivie de nouveaux déplacements relatifs devient la déformation dominante pour des contraintes fortes, et la phase où la destruction des grains se produit peut être déterminée par contrôle de la composition granulométrique.

La déformation élastique peut être définie assez facilement lors du déchargement de l'échantillon.

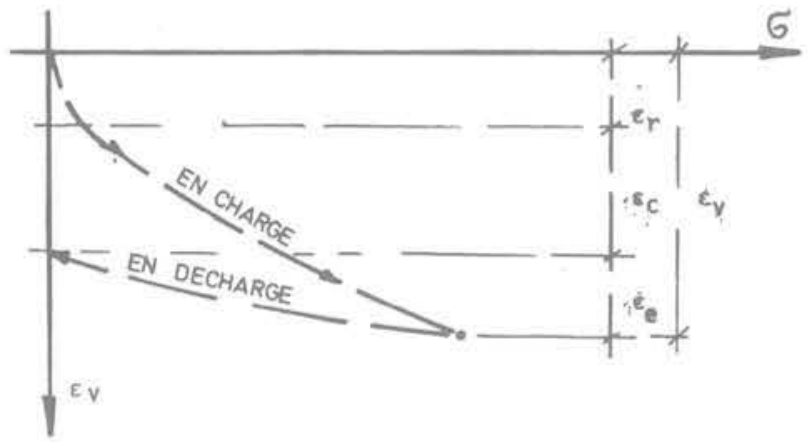

Figure 11

Eu égard à ce qui précède, la déformabilité dépend le plus de l'état initial du matériau et du degré de destruction des grains, c'est-à-dire de la grandeur des forces de contact provoquant la rupture. Pour la même contrainte moyenne, le matériau dont les grains sont les plus gros et dont la courbe granulométrique est la plus uniforme est le plus succeptible à l'effet de fragmentation.

Afin d'obtenir les grandeurs équivalentes de déformabilité pour les matériaux à gros grains (réels) et à petits grains (échantillons), il faut exercer des forces semblables aux contacts de grains, c'est-à-dire des forces provoquant des destructions et des déformations élastiques similaires.

D'après la solution de Hertz (1881-1895), le rapprochement relatif des centres de deux sphères élastiques est donné pour la relation :

$$
S=p^{2 / 3} \cdot A^{2 / 3} \cdot R^{-1 / 3}=\left(\frac{P^{2} A^{2}}{R}\right)^{1 / 3}
$$

où :

$P=$ force au contact de deux sphères;

$\mathrm{R}=$ rayon des sphères;

$\mathrm{A}=$ constante élastique.
Pour obtenir les déformations élastiques identiques $S_{\text {r }}$ et $S_{e}$ (du matériau réel et du matériau de l'échantillon) il faut que :

$$
P_{r}^{2 / 3} \cdot A_{r}^{2 / 3} \cdot R_{r}^{-1 / 3}=P_{e}^{2 / 3} \cdot A_{e}^{2 / 3} \cdot R_{e}^{-1 / 3}
$$

soit :

$$
\frac{P_{r}^{2} \cdot A_{r}^{2}}{P_{e}^{2} \cdot A_{e}^{2}}=\frac{R_{r}}{R_{e}}
$$

Pour $A_{r}=A_{e}$, on $a$ :

$$
\frac{P_{r}}{P_{e}}=\left(\frac{R_{r}}{R_{e}}\right)^{1 / 2}
$$

Si l'on pouvait réaliser des déformations élastiques égales dans des échantillons comportant le même nombre de grains et de points de contact et se distinguant par les dimensions des grains, le rapport des forces assurerait pour le matériau réel une force de contact toujours plus élevée :

$$
P_{r}=P_{e} \cdot\left(\frac{R_{r}}{R_{e}}\right)^{1 / 2}
$$

étant donné que $R_{r}>R_{e}$.

La destruction des grains sous l'action de forces concentrées d'après la solution théorique (Jaeger, Cook, 1968) pour le cylindre circulaire correspond à :

$$
\max \sigma_{\theta_{r}}=\frac{P}{\pi \cdot R} \text {. }
$$

La relation de similitude :

$$
\max \sigma_{\theta r}=\max \sigma_{\theta e}
$$

implique la condition :

$$
\frac{P_{r}}{P_{o}}=\frac{R_{r}}{R_{e}}
$$

indiquant que les forces sont proportionnelles au rapport des rayons.

Étant donné que la force limite de contact du grain réel est

$$
\mathrm{P}_{\mathrm{r}-\lim }>\mathrm{P}_{\mathrm{e} \cdot \mathrm{lim}} \cdot \frac{\mathrm{R}_{r}}{\mathrm{R}_{\mathrm{e}}}
$$

et que $P_{\text {r.lim }}>P_{e \cdot l i m}$ en déformation élastique, la destruction des grains ne se produit qu'après la réalisation de la déformation élastique équivalente à savoir un rapport des forces de contact différent de celui obtenu dans le cas de la déformation élastique.

On peut donc constater que le changement de la déformabilité en fonction de la grosseur du grain ne peut pas être exprimé par une formule théorique rationnelle, mais on doit :

a) Examiner plusieurs niveaux de contrainte avec la même composition granulométrique et déterminer les rapports des déformations non élastiques et élastiques.

b) Examiner avec des états de contrainte identique différentes compositions granulométriques, en modifiant tout d'abord uniquement la grosseur du grain et ensuite aussi la forme de la courbe granulométrique.

Toutefois des problèmes apparaissent aussi du point de vue de la réalisation des états de contraintes équivalents lors des essais sur échantillon de dimensions différentes essayés dans des appareils œdométriques de grande dimension.

Le premier problème représente l'influence de l'écrasement et de la fragmentation des grains qui se produisent aux contacts de l'échantillon et des parois métalliques. 


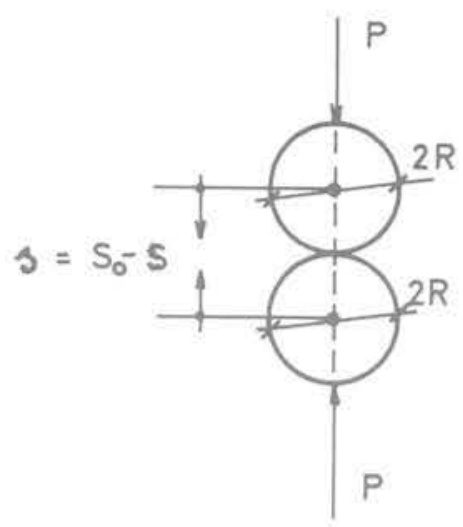

$s=S_{0}-S=\sqrt[3]{\frac{P^{2} A^{2}}{R}}$

$$
\begin{gathered}
\frac{R r}{R e}=\frac{P r^{2} \cdot A r^{2}}{P_{e}^{2} \cdot A_{e}^{2}} \\
A_{r}=A_{e} \rightarrow \frac{P_{r}}{P_{e}}=\left(\frac{R r}{R_{e}}\right)^{1 / 2}
\end{gathered}
$$

B) DANS LE DOMAINE PLASTIQUE (RIIPTURE)
A) DAHS LE DCMAINE ELASTIQUE

$$
\begin{aligned}
& \max G_{\theta}=\frac{P}{\bar{S} \cdot R} \text { (POUR ÉCHANTILLON CYLINDRIQUE) } \\
& \frac{P_{r}}{P_{e}} \geqslant \frac{R_{r}}{R_{e}}
\end{aligned}
$$

c) DANS LA COUCHE AU CONTACT AVEC SURFACE PLANE METALLIQUE

$$
\begin{aligned}
& \frac{\max q s p-g}{\max q g \div g}=\left(\frac{P s p-g}{P_{g-g}}\right)^{1 / 3} \text { si } E_{s} \gg E_{g} \\
& P_{s p-g}=(3 a) 4: \times P_{g-g} \longrightarrow \max Q_{s p-g} \equiv 1.5 \max q_{g-g}
\end{aligned}
$$

Figure 12
En comparant la solution de Hertz pour les contraintes maximales aux contacts d'une surface plane et d'une sphère d'une part et de deux sphères d'autre part on obtient:

$$
\frac{\max q_{s-g}}{\max q_{g-g}}=\left(\frac{P_{s-g}}{P_{g-g}}\right)^{1 / 3} \cdot\left(\frac{K_{g} \cdot R_{g}}{\left(K_{s}+K_{g}\right) \cdot R_{g}}\right)^{2 / 3}
$$

Si on appelle $Q$ les contraintes aux contacts et $P$ les forces aux contacts et en notant $(s p-g)$ pour l'enveloppe de l'échantillon aux contacts avec la surface métallique, et $(\mathrm{g}-\mathrm{g})$ pour les contacts entre les grains on obtient:

$$
\frac{\max Q_{s p-g}}{\max Q_{g-g}}=\left(\frac{\max P_{s p-g}}{\max P_{g-g}}\right)^{1 / 3} \cdot\left(\frac{K_{g}}{K_{s p}+K_{g}}\right)^{2 / 3}
$$

où les constantes élastiques sont :

$$
\mathrm{K}_{\mathrm{sp}}=\frac{1-\nu_{\mathrm{sp}}^{2}}{\pi \mathrm{E}_{\mathrm{sp}}} ; \quad \mathrm{K}_{\mathrm{g}}=\frac{1-v_{\mathrm{g}}^{2}}{\pi \mathrm{E}_{\mathrm{g}}} .
$$

Étant donné que :

on obtient :

$$
\mathrm{K}_{\mathrm{sp}} \ll \mathrm{K}_{\mathrm{g}} \text { car } \mathrm{E}_{\mathrm{sp}} \gg \mathrm{E}_{\mathrm{g}}
$$

$$
\max Q_{\mathrm{sp}-\mathrm{o}}=\max Q_{\mathrm{g}-\mathrm{g}}\left(\frac{\max \mathrm{P}_{\mathrm{sp}-\mathrm{q}}}{\max \mathrm{P}_{\mathrm{g}-\mathrm{g}}}\right)^{1 / 3}
$$

En supposant que chaque grain au contact avec la surface métallique transmet la force sur 3 à 4 grains avoisinants dans la masse, on obtient:

$$
P_{\text {sp }-\mathrm{g}}=(3 \text { à } 4) \cdot P_{g-g}
$$

d'où : $\quad \max Q_{\mathrm{sp}-\mathrm{g}}=1,5 \cdot \max Q_{\mathrm{g}-\mathrm{g}}$.

Dans l'essai de compressibilité la destruction des grains est donc environ $50 \%$ plus élevée dans la couche au contact avec les parois métalliques de l'appareil qu'à l'intérieur de l'échantillon. 
II apparaît que deux rapports sont nécessaires pour l'étude de similitude d'échantillons de différente grosseur et notamment

$\mathrm{A}=\frac{0 \cdot \mathrm{D}_{\max }}{\mathrm{V}}=$

Superficie de l'enveloppe $\times$ Diam. maximal du grain Volume de l'échantillon

ou bien

$$
\psi \cdot \frac{D_{\max }}{H}=\frac{\text { Diamètre maximal du grain }}{\text { Hauteur de l'échantillon }} \cdot \psi
$$

où $\psi$ est un coefficient dépendant du rapport du diamètre à la hauteur de l'cedomètre.

La figure 13 présente le résultat d'essais de compressibilité à l'œdomètre où la déformation verticale totale $\varepsilon_{z}$ est répartie en déformation provenant des actions de contact des matériaux avec les parois de l'appareil $\varepsilon_{0}$ et en déformation apparaissant dans la masse de l'échantillon $\varepsilon_{m}$, pour différents rapports

$$
\frac{0 \cdot D_{\max }}{V}=A(0,18 ; 0,30 ; 0,84) \text {. }
$$

Ces essais ont démontré que la grandeur $\varepsilon_{0}$ influence essentiellement la valeur $\varepsilon_{z}$, atteignant $50 \% \varepsilon_{z}$. II a été démontré aussi que pour une mêrne valeur de $\varepsilon$, la grandeur $\varepsilon_{z}$ augmente si le paramètre $\frac{0 \cdot D_{\max }}{V}=A$ augmente.

Pour les rapports géométriques standards des échantillons cylindriques à l'œdomètre $H=R / 2$.

On obtient la valeur :

$$
\begin{gathered}
\frac{0 \cdot D_{\max }}{V}=\frac{2 R \pi \cdot H+R^{2} \pi}{R^{2} \pi \cdot H} D_{\max }=\left(\frac{2}{R}+\frac{1}{H}\right) D_{\max } \\
\frac{0 \cdot D_{\max }}{V}=\frac{4 D_{\max }}{R}=\frac{2 D_{\max }}{H},
\end{gathered}
$$

soit

Plus le rapport $\psi \cdot D_{\max } / H$ est faible, moins l'influence de la fragmentation du grain près des parois se répercute sur les résultats; l'uniformité de la courbe granulométrique exerce aussi une influence car les matériaux de composition uniforme sont plus sensibles aux effets de fragmentation des grains près des parois.

Le deuxième problème est relatif à l'état des contraintes apparaissant au contact des bases supérieure et inférieure et des faces latérales.

Cette non-homogénéité apparaît aussi dans des cedomètres où l'on atténue le frottement sur les faces latérales étant donné que ce frottement provient aussi de la distribution des contraintes normales, et non seulement de la valeur du frottement sur la surface latérale de l'cedomètre.

Afin de déterminer les conditions de la prévision de la déformabilité, notamment pour la prévision de la grandeur du module de compressibilité à l'cedomètre, il faut:

a) Examiner dans le même œedomètre, pour plusieurs niveaux de contrainte $\left(\max \sigma_{y}\right)$, différentes courbes granulométriques et déterminer les déformations caractéristiques (provenant des déplacements relatifs, de la fragmentation et des propriétés élastiques des grains) et leur dépendance vis-à-vis du changement des contraintes max $\sigma_{v}$ et de la dimension maximale des grains $D_{\max }$ b) Dans des œdomètres de caractéristiques $\mathrm{H} / 2 \mathrm{R}$ différentes, déterminer l'influence que ces caractéristiques exercent sur les résultats, pour la même courbe granulométrique et le même max $\sigma_{v}$.

Si l'on utilise un grand échantillon cylindrique avec un chargement rigide axial et un chargement flexible radial, les essais sont limités à des contraintes normales relativement peu élevées pour les matériaux granulaires de formes anguleuses et pointues (pierre concassée et cailloutis).

L'influence de la fragmentation est moindre, localisée uniquement sur les bases supérieure et inférieure de l'échantillon. Mais la mesure de la déformation volumétrique de l'échantillon est plus difficile ainsi que la détermination de la courbe effort déformation (chargement et déchargement).

En raison de maintes difficultés pratiques de manipulation lors de la mise en place de l'échantillon dans une enveloppe flexible, ce mode de détermination de la déformabilité n'est pas utilisé dans les essais de routine.

\section{Conclusions}

Malgré la complexité évidente des propriétés de déformation et de résistance au cisaillement des matériaux à gros grains et des grandes difficultés rencontrées lors de leur détermination expérimentale, des possibilités réelles et pratiques existent pour l'exécution d'essais appropriés en laboratoire permettant de déterminer des valeurs fiables de ces propriétés. Au niveau actuel des connaissances des processus d'interaction entre les grains, on peut déterminer des relations entre le comportement général du matériau et certains paramètres relatífs à la grosseur de grains; à la forme de la courbe granulométrique ou au niveau des contraintes.

Les applications pratiques nécessitent la connaissance qualitative de ces relations afin d'éviter des erreurs dans les essais utilisés pour déterminer les propriétés globales sur la base des facteurs suivants :

a) Composition pétrographique identique (qualité des grains).

b) Courbe granulométrique semblable.

c) Conditions identiques au point de vue de la dépense d'énergie provoquant le changement de volume.

d) Conditions identiques au point de vue de la dépense d'énergie produisant la fragmentation des grains.

e) Rapport correct entre les dimensions des échantillons et celles des grains.

f) Conditions semblables au point de vue de la porosité initiale.

La figure 15 présente le schéma des paramètres principaux de l'analyse élémentaire, en majorité de caractère qualitatif, ainsi que les paramètres de l'analyse globale, de caractère quantitatif. II existe encore une incertitude supplémentaire des caractéris tiques réelles du matériau engendrée par la mise en place lors de l'exécution et du fait des variations possibles en cours de travaux. Cette indétermination impose la nécessité d'envisager une marge d'incertitude pour les propriétés du matériau réel devant comprendre les modifications des moyennes espérées jusqu'aux valeurs possibles extrêmes des paramètres de densité et de déformabilité. 

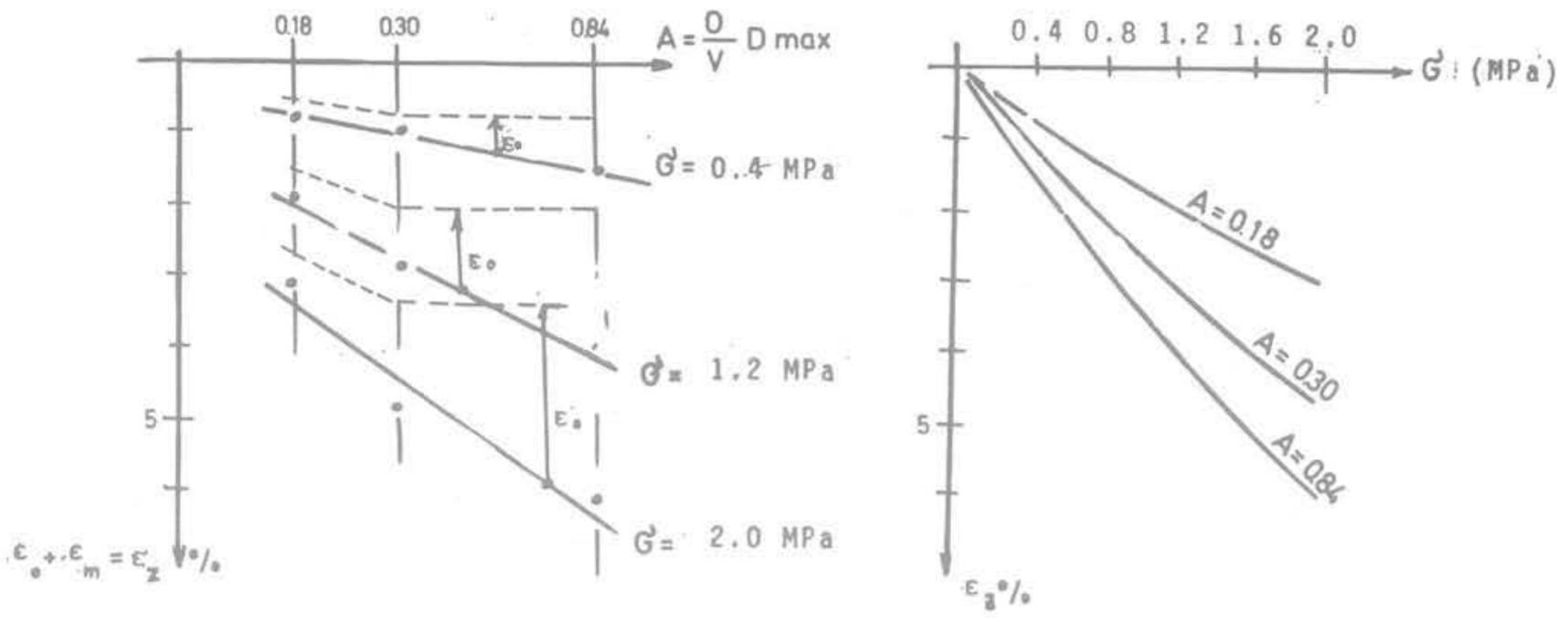

M. POPOVICH - 1978. SARAJEVO)

Figure 13

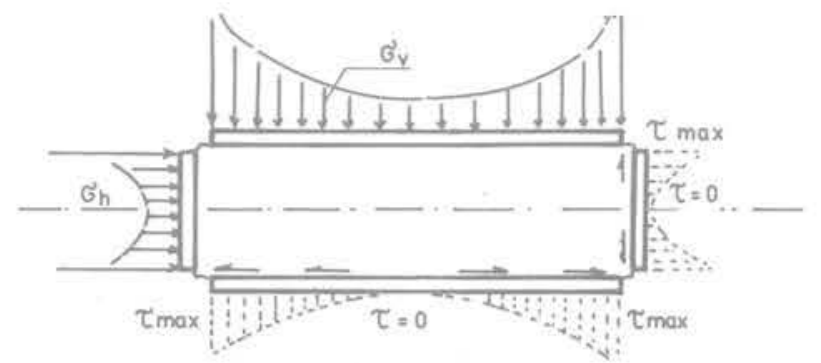

Figure 14

LES ESSAIS A L'ECHELLE DE L'ECIIANTILLON

\section{ANALYSE ELEMENTAIRE}

GRANDEUR, COMPOSITION

GEOMETRIE, QUALITÉ ET ASSEMBLAGE DES GRAINS

INTERACTION CINEMATIQUE

INTERACTION STATIQUE

POROSITÉ - DENSITÉ

\section{ANALYSE GLOBALE}

DILATANCE

NIVEAU DE CONTRAINTES

VOLUME REPRESENTATIF

DEFORMATIONS IRREVERSIBLES

FRAGMENTATION DES GRAINS

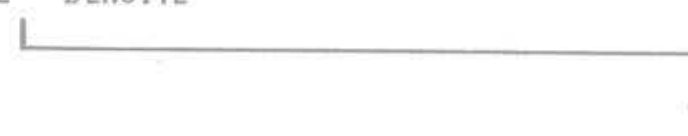

LES PROPRIETES MECANIQUES

A L'ECIIELLE REELLE

$\downarrow$

AVEC INCERTITLDES AVANT L'EXECUTION

- COMPOSITION GRANULOMÉTRIQUE

- DENSITÉ REELLE

- QUALITÉ DES PLUS GROS GRAINS

$\frac{1}{\nabla}$

VALEURS EXTREMES ET MOYENNES

DES PARAMETRES

Figure 15 
En maintenant certains paramètres constants et en faisant varier les autres, on peut apprécier l'influence de ces changements et constater la plage d'influence sur les paramètres de résistance et de déformabilité. C'est l'approche la plus efficace et la plus correcte de ces problèmes, d'autant plus que les variations de la composition des matériaux à gros grains sont souvent considérablement plus grandes que celles des matériaux à petits grains.

II est donc plus correct de déterminer d'abord les limites des variations possibles des paramètres de déformabilité et de résistance au cisaillement pour ensuite décider de leur application dans les calculs.

La figure 16 présente un schéma pratique pour la programmation et pour la détermination des propriétés des matériaux à gros grains.

Pendant la première étape les conditions envisagées pour le matériau réel doivent être déterminées. Le problème le plus complexe est celui de la détermination de la "qualité du grain", ce qui sous-entend les propriétés de surface, de forme, de déformabilité et de densité. Des recherches très étendues doivent être faites sur ce sujet.

Pendant la deuxième étape la dimension des échantillons à soumettre aux essais doit être choisie en tenant compte de la nécessité de déterminer aussi l'influence de l'échelle de l'échantillon par rapport aux matériaux expérimentés.
Aux cours de la troisième étape on effectue la mesure des propriétés des matériaux expérimentés tout en faisant varier certains paramètres élémentaires.

Pendant la quatrième et cinquième étapes, on détermine les propriétés cherchées dans les conditions envisagées par le matériau réel, par essais sur des matériaux de grains plus petits, et on procède par corrélations en minimisant la sensibilité à la modification des paramètres élémentaires.

Les observations faites durant la construction et lors de l'exploitation des ouvrages réalisés offrent la possibilité de vérifier certains paramètres calculés et en particulier ceux relatifs à la déformabilité des matériaux. Pour effectuer une vérification précise, il faut connaître la qualité des matériaux mis en œuvre au point de vue de la composition granulométrique, de la compacité et de la teneur en eau du phénomène de ségrégation, de la composition pétrographique des grains, de leur forme, de leur sensibilité à la fragmentation, des changements de qualité (ramollissement, écrasement), etc. A ce stade il est nécessaire d'établir des paramètres pratiques de classification et de grouper les matériaux à gros grains en classes principales pour lesquelles on peut envisager plus facilement des valeurs approximatives des paramètres de déformabilité et de résistance et effectuer le dépouillement des données existantes.

\section{PROGRAMMATION PRATIQ'JE}

1) ESTIMER LES LIMITES DES - NIVEAUX DE CONTRAINTES

- GRANULOMETRIES

- DENSITES

- QUALITES DES GRAINS

(A L'ECHELLE REELLE)

2) SELECTIONNER LES DIMENSIONS DES ECHANTILLONS : LES PLUS GRANDES SELON LES POSSIBILITES DU LABORATOIRE OU DU CHANTIER

3) ETUDIER LA RELATION ENTRE LE COMPORTEMENT GLOBAL (DILATATION, FRAGMENTATION DES GRAINS, DEFORMATIONS IRREVERSIBLES, ETC) ET LES PARAMETRES ELEMENTAIRES (GRANDEUR ET COMPOSITION DES GRAINS, POROSITE, ASSEMBLAGE, ETC)

4) ESTIMER .IES VALEURS MOYENNES ET EXTREMES, A PARTIR DE L'ECHELLE DES ECHANTILLONS ET DES RELATIONS QUANTITATIVES

5) EXTRAPOLER LES PROPRIETES POUR LES CONDITIONS REELLES

6) REALISER DES OBSERVATIONS DANS LES CONDITIONS REELLES PENDANT LA CONSTRUCTION ET PENDANT LE. FONCTIONNEMENT DE L'OUVRAGE 


\section{Référence bibliographique}

P. ANAGNOSTI, Shear strength of soils other than clay, General report, Session 3. Europ. Geotechn. Conf., Osio, 1967.

P. ANAGNOSTI, Design parameters for fills.

General report, Session 6. Europ. Geotechn. Conf., Brighton, 1979

A. W. BISHOP, The strength of soils as engineering materials.

Geotechnique, vol. 16, 1966.

R. J. FROST, Some testing experiences and characteristics of boulder-gravel fill in earth dams.

ASTM Symposium, Los Angeles, June 1972

J. C. JAEGER, N. G. W. COOK, Fundamentals of Rocks mechanics.

Chapman and Hall, London, 1976.

T. M. LEPS, Review of shearing strenght of rockfill. Journ. SMFD, Proc. ASCE, July 1970.

M. MAKSIMOVIC, Limit equilibrium for non linear failure envelope and arbitrary slip surface.

III, ICNNG, Aachen, April 1979.
N. D. MARACHI, C. K. CHAN, Evaluation of properties of rockfill materials.

Journ. SMFD, Proc. ASCE, January 1972.

R. J. MARSAL, Mechanical properties of rockfill.

- Embankment-Dam Engineering, Casagrande

Volume, John Wiley and Sons, 1973.

- 12th ICOLD, Mexico 1976, Q44, R10.

M. POPOVIC, Scale effects at compressibility and direct shear tests on coarse-grained materials.

Publications of the Institute of Geotechnical and Foundations, Sarajevo, Yugoslavia, vol. 6, 1978.

P. W. ROWE, The stress-dilatancy relation for static equilibrium of an assembly of particles in contact.

Proc. Royal Society, London, vol. 269, 1962.

C. SADASIVAN, V. S. RAJU, Theory for shear strength of granular material.

Journ. GED, Proc. ASCE, August 1977. 
